\title{
Video Assessment Tasks in Tertiary Education: Practice and Experience in STEM
}

\author{
Matthew Roughan \\ ARC Centre of Excellence for Mathematical \& Statistical Frontiers (ACEMS), \\ School of Mathematical Sciences, University of Adelaide, Australia \\ matthew.roughan@adelaide.edu.au
}

\begin{abstract}
The COVID-19 (Coronavirus) pandemic mandated many changes in delivery and assessment in the first half of 2020. This paper describes one tool for assessment that might never have been trialled without this impetus - video creation - that proved to be a valuable and interesting approach with many advantages over traditional assessment methods. Used in conjunction with traditional assessments I believe this will enhance both quality of assessment and student engagement ${ }^{1}$.
\end{abstract}

\section{INTRODUCTION}

"Who dares to teach must never cease to learn." John Cotton Dana

This semester, we were forced to make many changes to teaching courtesy of the COVID-19 pandemic. A great many courses were changed; I wish to discuss one course in particular: Advanced Mathematical Perspectives 1 (AMP1). The lessons learned seem to be quite universal, and should apply over a range of STEM disciplines.

AMP1 is a course taught to an elite group of mathematics students as part of an "Advanced" degree, one of a set of degrees that the University of Adelaide offers to highperforming students. It is promoted as more challenging and hence more interesting than a traditional degree. It is aimed at enhancing employment prospects for students who complete it, as well as enabling them to get a jump start into a research career if this is their chosen direction. This paper concerns the 1st year (freshman) Advanced subject, which is given a generic name because its topic/content may vary from year to year depending on the team teaching it. This year the topic was Information Theory.

This white paper is concerned with one element of the assessment of this subject, which in previous years was a conference-style poster presentation. COVID-19 restrictions made this impossible and so, much as in many actual conferences [For20], the poster presentations were replaced by a video presentation.

1. An earlier version of this paper appeared as a white paper at the ACM Sigcomm Education Workshop and Online Discussion, 2020. http //gaia.cs.umass.edu/sigcomm_education_workshop_2020/index.html
This was a very new form of assessment for us, and seems to be unusual, though not unprecedented, in university education and hence worth reporting.

Key insights were:

- Video presentations have some of the advantages of an oral exam, e.g., they are representative (plagiarism resistant), encourage deeper learning [ICR20], and are a more authentic form of assessment than a traditional exam, but importantly one of the serious disadvantages of an oral exam - the stress on students and examiners - is minimised. Likewise English language difficulties can be more easily addressed when students have time to prepare a script rather than when they have to communicate on the fly.

- Video creation engaged the students by involving them in an activity they see almost omnipresently in current society.

- Content coverage is poor (a short video can only really touch on one topic) and so such assessment should be coupled to more traditional assessments.

Overall the experience of using this form of assessment was overwhelmingly positive, and I intend to continue to use it in this and other subjects even when COVID-19 restrictions are relaxed, and wish to encourage its adoption elsewhere.

\section{BACKGROUND}

\subsection{Related Work}

There is some informal advice and commentary on the use of videos as an assessment exercise: see for instance [CAL19], [Ale19], [wat20], but there appears to be only a small formal literature on video assessment exercises, little of which is oriented at quantitative subjects such as mathematics or computer science ${ }^{2}$. One instance in the formal literature [FDWT16] considers the use in accounting, primarily to promote soft skills (communication, project management, ...), which are of increasing importance in almost all workplaces.

2. It is possible there is a larger literature that is hard to discover because most searches return a large list of items either on using videos as a means to deliver content, or videoing classrooms as a means to assess and improve teacher performance. 
While we seek to teach these skills here, these are not the only benefits of such exercises.

We can learn about other benefits by looking at the much larger body of work on oral examinations, which have characteristics in common. There are several advantages to oral examinations:

- Oral exams present a variation in a typically uniform set of assessments in mathematics (dominated by written solutions to questions). Such exams require more creativity from the students than common assessment tasks, which can often be completed using imitative reasoning [ICR20], [HCW10].

- Students perceive oral exams as requiring conceptual understanding over memory with a correspondingly positive impact on their learning strategies [ICR20].

- Oral exams avoid some of the difficulties introduced by written communication [ICR20] — some students can convey their understanding better in the oral context.

- Oral exams are more authentic than exams, in that they more accurately represent realistic exchanges in the workplace (job interviews, or other forums where ideas must be communicated and defended) [HCW10].

- Oral exams can be more inclusive [HCW10] towards students with disabilities (particularly those such as dyslexia).

- Oral exams are plagiarism resistant [HBR20], [HCW10] as students convey their understanding using their own words.

Preparing a video assessment has some of these advantages but also avoids the disadvantages of interrogative oral exams. Iannone et al. [ICR20] found that students still prefer closedbook written exams over oral exams, notable reasons being related to anxiety over pressure of answering questions with someone looking over your shoulder [ICR20], [HCW10], and that they favour extroverts [HCW10] ${ }^{3}$.

Video creation removes or reduces these problems by giving students time and space to prepare without concern over immediate impact of errors or stress-related problems. It is reminiscent of the oral assessment procedure presented by Jensen [Jen10], where students are given (limited) time to prepare a short, oral presentation on a topic revealed at the start.

The approach also has the advantage of engaging the student actively in their assessment.

Ultimately, the idea of using oral communication reflects the position of Plato (and many subsequent philosophers) giving primacy to the spoken rather than written word as the means for a person to genuinely communicate their identity and ideas. A video is just the most modern take on this idea.

\subsection{Advanced Mathematical Perspectives 1}

Students in the Advanced degree share most of their coursework with traditional Bachelors students, the primary

3. I have not found it mentioned anywhere in the literature, but on a personal note I also find that administering an oral exam creates stress for the examiner who is under pressure to perform consistent and objective assessment in real time. You cannot go back (in time) during an oral examination process to correct or refine previous assessments in the light of later presentations in the way you can with written exams. differences being:

1) the course is hard to gain entry to;

2) students must maintain a credit grade point average; and

3) each year students have a compulsory "Advanced" course ( 3 units of 24 for each year).

The subject "Advanced Mathematical Perspectives 1 " is intended to challenge and expand the skills of this elite group in their first year at university.

Apart from simply providing these students with a challenge, the subject has wide set of goals:

- introduce the students to the different branches of the mathematical sciences: pure, applied and statistics;

- teach the students research skills (including the ability to communicate their work through standard means, traditionally through a report written in LaTeX and a oral poster presentation);

- allow the students to explore a more realistic application than is possible in a traditional 1st year undergraduate mathematics course;

- support and develop "side-skills, " e.g., presenting mathematical arguments, working with data, using computer code to implement their ideas, etc.; and

- to meet and form a community with their peers.

The course has been, since its inception, taught as a flipped classroom. Students are presented with a minimal amount of formal course material and expected to research a larger body with guidance. In 2020 this formal material took the form of approximately 10 hours of videos specifically recorded for this course in the COVID-19 environment, plus associated notes and handouts.

In parallel, the majority of the students are also studying standard 1st year undergraduate courses on Mathematics (Calculus and Linear Algebra); computer programming (Matlab, C and R); and an elective subject (which varied widely amongst the students even to subjects such as English Literature and Music). Ideally, the advanced course is synergistic with these, even the electives.

This year's cohort of students was 27 strong. A third of the students were female. There are widely reported problems in gender equity in STEM subjects, however this percentage is consistent with undergraduate recruitment in the mathematical sciences in Australia; the number of strong female candidates, while not proportionate, is not as biased as in some areas of STEM [AMS17]. Nevertheless this should be improved, as should the rate at which this translates to research careers in mathematics. A key factor is ensuring a high quality experience for all students, both to encourage new enrolments and to retain students in STEM careers. Hence, this course must encourage a diversity of students, not just those stereotypical of STEM careers.

It is also important to note that between $10-20 \%$ of students in this subject in recent years have a reported disability, most often related to problems in reading and writing (e.g., dyslexia) or anxiety, and supporting these students is also very important (particularly in a year of higher than normal stress resulting from COVID-19).

There were many changes to the course to meet the challenges of this year, but this report primary considers the issue of assessment. 


\section{Assessment Principals and Goals}

\subsection{Assessment Goals}

In AMP1 we aim to assess student abilities consistent with the goals of the course, such as their ability to:

- $\quad$ conduct research, i.e.,

- to find and understand source materials (written literature on a topic);

- to perform analysis (statistical or otherwise);

- to work with data;

- develop arguments using high-level reasoning and critical thought; and

- communicate results.

The assessment also aims to cover topics from Pure Mathematics to Applied Mathematics and Statistics.

To these ends the students are asked to create two projects: one on a Pure Mathematics topic, and the other combining Applied Mathematics and Statistics. The Pure project (this year) was conducted separately before COVID19 restrictions were enacted and so this report concerns the Applied/Statistics project.

\subsection{Assessment Tasks and Weighting}

Students assessment tasks and their weight in the final assessment were originally determined by the School's Learning and Teaching Committee, and historically they were weighted as follows:

- a written report on their Pure Mathematics project $(25 \%)$;

- a written report on their Applied/Statistics project $(60 \%)$; and

- an oral poster presentation on their Applied/Statistics project (15\%).

The weighting towards Applied Mathematics and Statistics was not determined by perceived importance, but rather because this component was required to touch on a wider range of topics (in addition to Statistics, the Applied component was expected to include aspects of Probability, Optimisation, Calculus and/or Linear Algebra). The written report this year was the same as in previous years. The component that varied was the oral presentation.

\subsection{Punished By Rewards and Self-Guided Projects}

It is now recognised that assessment may, in itself, cause problems in students' learning [Koh99]. Grades become the goal not the measure of education. For instance:

"With education now a 'business' and degrees sold as a 'product' - there is less connection and understanding that a university is about acquiring knowledge." Bretag, 2018

Simple impacts are that students (who are very aware of risk) will choose less challenging (and hence educational) topics and activities [Har78]. Moreover, grades can create unhappiness. Surprisingly this is not just about bad grades. Even students who routinely receive high grades are less happy and engaged as a result of grading [Koh99], [Har78].
We cannot, at this juncture, give up on grades entirely: one of the functions of a modern university is to certify the skills and knowledge of their graduates. However, there are means to mitigate the negative affects of grading. One of these is to give students greater control over their assessment, for instance by giving them control of the topic of their projects.

As a side-reward, it is much more enjoyable for an examiner to read or listen to presentations on 20+ diverse topics than to hear the same topic presented many times, and thus helps the assessor maintain focus.

\subsection{Ensuring Representative Assessment}

The modern world presents many challenges for fair and accurate assessment. One serious concern is the rise of online cheating-support sites where (i) support is given to answer specific questions (e.g., Chegg ${ }^{4}$, Slader ${ }^{5}$ or Stack Exchange ${ }^{6}$ ) or (ii) help to perform assessment can be purchased (e.g., My Math Genius ${ }^{7}$ ), i.e., contract cheating. At a deeper level it can be hard for students to judge the difference between collaboration (i.e., students helping each other learn, which we encourage) and collusion or copying (which are considered cheating).

In the world of mathematics and computer science dishonest academic behaviours are particularly problematic because in many cases it is impossible to detect students who have copied a solution or otherwise obtained illicit help because there is one "correct" solution and so we might expect many students to hand in nearly identical work. Moreover in many cases it is easy to modify an answer to look different by simple substitution of a set of symbols or names. Hence plagiarism detectors do not work. Detecting specific cheating is often possible only where students copy incorrect work and thus have uniquely identifiable mistakes in common. Experience with, e.g., Chegg, is that solutions posted there often contain such mistakes (accidental or deliberate), but attempts to cheat are still damaging to student learning.

A closed and invigilated exam in which student identities are checked and invigilators personally supervise students to ensure that the work they present is their own is the standard approach to prevent attempts to cheat despite many concerns with traditional exams such as the manner in which they create a gender penalty $\left[\mathrm{SCA}^{+} 19\right]$.

However, in-person exams were prohibited this year. Most mathematics subjects involved a substantial online exam component, and in many cases direct attempts to cheat were found (e.g., questions posted on Chegg within an hour of the start of the exam). Tracey Bretag, perhaps Australia's foremost expert on academic integrity, also claims that more cheating goes on even in traditional invigilated exams than we would expect [HBR20] - contract cheating is pernicious and hard to detect.

The Advanced subjects, however, have never used examinations as an assessment method. Invigilated exams are fraught with many other issues. Notably they increase stakes for students inducing anxiety, and their results are a poor proxy for genuine mathematical ability as they can often be

4. www.chegg.com

5. https://www.slader.com/.

6. math.stackexchange.com.

7. www.mymathgenius.com. 
completed through imitative reasoning [ICR20] rather than genuine creative problem solving.

Instead, AMP1 has asked students to prepare two written project reports (one on a Pure Mathematics topic, and one on a joint Applied-Statistics topic); and to create an oral poster presentation. It is still possible to "cheat" in such exercises by purchasing or otherwise obtaining illicit help in preparing said assessment items. However the incidence of cheating is thought to be very low because

- personalised assessments discourage cheating [HBR20];

- students work closely with their academic facilitators throughout the semester in developing their ideas, and so overt cheating would result in a discrepancy between their in-person performance and final result; and

- the oral presentation is made in their own voice, so while they might have help in, for instance, constructing a script they must take ownership of the final product. And evidence also support that oral assessments discourage cheating [HBR20].

\section{Video Presentations}

Oral presentations have always been an important component of AMP1 assessment. Most obviously they test and promote oral communications skills, which are critical in the modern work environment. Not quite so obviously, but equally important, is the fact that explaining an idea requires that one understand it at a deeper level [ICR20], [HCW10], [Jen10].

Historically, the oral presentations in AMP1 were in a poster-session format. Students created an A1 $(594 \mathrm{~mm} x$ $841 \mathrm{~mm}$ ) poster with a very wide degree of latitude in style and content, and they were presented together with a short (5 minute) oral presentation in a large meeting room. Students were asked to browse other students posters, and senior members of the school were invited also, so the students had multiple opportunities to present their poster.

This type of large in-person event was not possible (in mid-June 2020) due to South Australian COVID-19 restrictions (based on the best advice about safety of staff and students). It might have been replaced by online presentations, but these would have lost most of the excitement of a poster session. Hence it was decided that the the students would create a "micro" video presentation.

\subsection{Why a Video?}

There is some informal advice and commentary on the use of videos (or screen-cast) as an assessment exercise: see for instance [CAL19], [Ale19], [wat20] but there is little formal literature. However we can draw from our understanding of other assessment exercises to make some general comments:

- Explaining an idea requires deeper understanding than simply being able to use it to calculate a number.

- Thinking about an idea in a different way from usual prompts different learning channels in your brain, enhancing retention.
- Creating videos is a useful skill. It is becoming a major means of delivering to various forms of clients. It is a useful, transferable, communications skill.

Additional considerations include:

- It is potentially a fun activity [CAL19, Ray], but at a minimum it is a good deal less intimidating than a real-time online presentation. For instance, mistakes can be edited when preparing a video.

- Modern students are very familiar with the short video format as they have grown up with YouTube et al., and many have already created their own videos [FDWT16]. Allowing students to express themselves through tools similar to those that they employ from day to day is more likely to engage them.

- The time taken by the students to create this replacement exercise should not be dramatically different from the original (i.e., creation of a poster and preparation for their short oral presentation).

Finally, I want to become better at this format for teaching. As noted above, there is no better way to learn a topic that to teach it. As it happens, I learned a lot about how to improve my own content videos from (i) working out how to formalise the task; (ii) explaining the concept to the students and answering their questions; and (iii) from watching their videos some of which were undoubtedly better than my own efforts.

\subsection{When?}

According to [SD20] by April 2nd, 2020, 194 countries had closed education institutions nationwide, affecting $91.3 \%$ of the world's population.

The University moved to online delivery mode around the 22nd of March (slightly later for some courses), which is the 4 th week of the 1 st semester of the year (the teaching component of the semester ran from March 2nd until June 12th). Hence the semester was already underway when COVID-19 changes were mandated. Changes to assessment (including the video component) where announced to the students as early as possible (the 25th of March). The assessable work for this component of the course had not been commenced at this point (the Applied and Statistics component of the course only commenced on the 23rd of March), so the students had not sunk time into obsolete tasks.

Detailed information on the video task was provided on the 23rd of April (during the mid-semester break). Students were asked to provide feedback on the proposed changes. Only a few students commented, and none specifically about the video. However many questions were asked over the next 6 weeks, and the exercise was refined in response to these questions.

Deadlines for the assessment were extended as much as possible (until June 22nd) to allow for students to comfortably undertake the new task.

\subsection{How?}

The essence of this activity was to provide some structure but allow for a great deal of freedom of expression. So some notes were provided on what was expected, but very few limits were placed on the activity. 
Students were required to make a short ( $\leq 3$ minute) video on a topic relevant to the course. It had to be their work, and they had to be present in the video, at least as narrator.

Otherwise they were given freedom. Technology, style and format were left up to them, with some suggestions given as help to get started.

Several pages of extra information were provided with helpful tips as well as a few useful links. However the expectation was (and this proved to be true) that the students would have some familiarity with and preferences for technology already. Thus mandating a particular choice would have been counter-productive.

The associated documents provided to the students also provided some advice about how the videos would be assessed. This was useful both in forming my own ideas about the project and in advising the students about what would be important in their video in terms of marks. A roughly $50 \%$ weight was given to the quality of the presentation and to content (see the following section for more details).

Apart from topic videos I prepared for the course, there was one additional exemplar provided by Dr Jonathan Tuke in the form of a stop motion introduction to calculating marginal distributions ${ }^{8}$.

Another important facet was providing the students with support [CAL19, Ray] as they worked on their projects and prepared the video. Of the 27 contact hours allocated to the Applied Mathematics and Statistics component of AMP1, more than half were allocated towards informal Zoom meetups to discuss the project, make suggestions and answer questions.

In addition Piazza was used to create an online discussion board which was used frequently once students bought into it (there was a start-up lag but after by the end of the course there were over 150 postings, between 27 students).

\subsection{Marking}

A good assessment task should have the following features:

- Validity: can we tell if the students learned what they were supposed to learn?

- Discrimination: it should separate the students by capability.

- Consistency: two equivalent items for assessment should get the same mark (often this is called reliability).

- Fairness: students with equivalent facility in the subject should have an equal chance of doing well. This is not consistency, as it should take into account how diversity of background may impact students in completing the task.

- Transparency: students can understand their mark.

It is not easy to achieve all of the features. In particular a video task has an inherently subjective component to its marking, tempering its ability to be marked consistently. However, we can improve consistency by providing a rubric describing the features of the video which will be marked. A number of existing rubrics for assessing video assessments

8. https://www.youtube.com/watch?v=CKmEogznGLs were considered [CAL19], but most were deeply mapped to a particular set of (non-mathematical) content and so an alternative was developed.

The resulting set of guidelines provided to the students, included specific advice about the assessment expressed in the acrostic: CLINIC RAID:

- Content: is the content correct?

- Length: is it shorter than the allotted time?

- Introduction: is it clear who you are and what the topic is?

- Narrative: does the video have a clear "story"?"

- Imagery: are the images and video clear? Is the lighting OK? Is it framed well? Is the camera steady?

- Clarity: is the explanation clear?

- Relevance: is the video relevant to the course?

- Audio: is it audible? Is there any background noise interfering? ${ }^{10}$

- Inclusivity: does the video talk to the right audience $^{11}$ ?

- Degree of difficulty: some topics are harder than others. Some extra credit was given for those who aimed at something harder.

These were not intended as a checklist or formal rubric so much as a means to reinforce the idea that presentation and communication skills were as important in this task as the specific content.

In addition throughout the course I emphasized that the goal would be to reward students for success, not to punish them for details. Getting details right in mathematics and computer science is important, but in a short video one cannot state everything that one might wish to in order to be completely precise. Students needed the freedom to present ideas at a higher, more abstract level.

One additional difficulty that always attends oral presentation assessments is that students whose first language is not English have an extra degree of difficulty. Although the University of Adelaide uses English as its teaching language, and students are expected to learn and communicate in English, it can be unfair to overly focus on this aspect of their work. This does not mean that correct use of English should not be assessed, but it is important not to be too critical of awkward phrasings, or speech that seems overly rehearsed, or emphasis that is not perfectly placed. The quality being assessed is communication not fluency.

\section{Results}

\subsection{The Videos}

The videos created by the students were wonderful! I judged that a more than one third were better than my own nascent efforts to create educational videos. The results are so good I obtained student permissions to use their videos not just as exemplars for future student videos, but also:

9. See [CAL19, Thorn].

10. Issues such as sound quality seem to have been a problem in some past examples of such assessments [CAL19, Roberts and Diggele].

11. The suggested audience was their classmates. 
- $\quad$ as exemplars to show my colleagues (many of whom are, like me, just starting to learn how to produce educational videos for the online teaching environment); and

- $\quad$ as actual teaching materials for future students.

The worst thing that could be said is that some videos were a little mundane. Most students chose topics from a provided list of examples with only a few venturing further or deeper, but they did approach the same topics quite differently.

There were other small glitches - a typo here or there, e.g., "Baye's Theorem" instead of "Bayes' Theorem," a few that ran slightly over 3 minutes, and there was the occasional misspoken word. However, it was hard to find serious fault in the large majority of videos.

Most students chose to narrate (without themselves in frame) across either a set of slides, or hand-drawn pictures. Some annotated slides as they narrated. However there were many variations: one student even did their own animation.

\subsection{Participation}

Four students out of a class of 27 dropped out of the course; two before any significant changes were announced, and a third citing "I am really not coping well with the current situation and I will be unable to submit the current assignment [not the video]. ." Even in a typical year, AMP1 is a challenging course both because of the difficulty of the material (which would typically not be presented to early undergraduate students) and the open-ended nature of the work expected. Unfortunately a small number students drop the course each year, even without the added stress of COVID-19. There is no indication that the video task was the specific cause for these students to drop the class.

\subsection{Student Experience}

Several students provided direct informal feedback during the semester in response to me asking their thoughts about my ideas for the course in the wake of COVID-19, or unprompted upon completion.

Only one comment was made specifically about the video exercise:

"Making the video was actually quite a fun task, I found it more enjoyable than an oral presentation, and it's always nice having the option to edit typos and make refineries."

Other comments were not specifically aimed at the video assessment exercise but certainly support the students' appreciation of the course modifications. For example:

"I like the sound of the new assessments you have proposed."

"I just wanted to say that not only will this style of teaching/marking encourage us students to take more risks and be more proactive in our learning, but I also wanted to say thank you."

"Thanks so much for your efforts in teaching the course with especially the difficulties present with this semester of teaching! It was amazing."
"Thank you for re-working the course to fit the online environment. I found it very intuitive amongst a sea of confusion."

"Thanks again for all your efforts this semester in ensuring that the class remained engaging and interesting online. Although I found it challenging, I really enjoyed it - I'd go as far as saying it was my favourite class this semester. Really made me excited to be doing a mathematics degree."

The University of Adelaide conducts Student Experience of Learning and Teaching (SELT) surveys after each course. Participation in such surveys is voluntary and typically very low (students are wildly over-surveyed, and consequently only participate when they have something they wish to specifically say about a course). However, this year there 16-19 answers (of 24) for each question. Unfortunately, the questions are standardised, so there is no specific feedback about videos here, and these conflate both Pure and Applied/Stats projects. However, the comments provided in the SELTs reinforce the voluntary comments above, for example:

"His [MR] transition online was very well done."

"He [MR] has adapted to online teaching really well and has always asked for feedback, and as far as I know, responded to that feedback to improve the course. His friendly interactions with us as aroup have also made the course really enjoyable."

"The best aspect of this course was the introduction to open based projects and research which I was not very used to at the start of the semester. The course has also taught me very useful skills to do with mathematics that I was able to effectively apply to my other courses."

"Favourite course of the semester."

"The transition online was well planned and plenty of resources were provided. The additionally resources and assistance was very good."

There were no specific negative comments about the video exercise (the majority of negative comments concerned the desire for more face-to-face interaction, which was understandable, but unavoidable in the circumstances).

The ratings provided by the students were also strongly positive, but hinted at one way in which the exercise should be improved. A sample of those ratings most relevant is included in Table 1.

\begin{tabular}{l|rr} 
Question & AMP1 & University \\
\hline $\begin{array}{l}\text { Overall, this course is intellectually stim- } \\
\text { ulating. }\end{array}$ & 6.4 & 5.9 \\
$\begin{array}{l}\text { This course includes digital activities } \\
\text { and resources that help me learn. }\end{array}$ & 6.3 & 5.8 \\
$\begin{array}{l}\text { The assessment tasks in this course help } \\
\text { me learn. }\end{array}$ & 5.7 & 5.9
\end{tabular}

TABLE 1. Average SELT survey results for AMP1 compared against the University averages across all courses. All scores are on a 7 point scale.

The most negative rating was to the question "The assessment tasks in this course help me learn." It appears that the students did not regard creating a video (and other tasks) as learning tasks. This may be because they contrast so completely with traditional tasks in mathematical 
sciences. There is a definite perception from many students and teachers that learning tasks in the mathematical sciences must be painful. However earlier feedback could also aid in perceptions of the educational value of such tasks. It would clearly have been valuable to students to receive feedback on a "first draft" video.

\subsection{Validity}

This assessment exercise was not intended as a formal experiment and was not conducted in a way that can provide conclusive, objective results about the validity of the assessment. Ideally such an experiment would be designed in advance with suitable controls and statistical design to ensure a large-enough sample. Informally it appeared to be successful. It appears easy for an assessor to judge the nature and quality of a students understanding from such a video. In addition to the quality of their narrative, the short length forces a student to focus on what is important, and in making those choices they display their understanding.

\subsection{Efficiency}

An important question in designing assessment is "How efficient is it?" We can dream up many assessment strategies, but in an environment where we are being asked to do more with less, we must concern ourselves with the cost of assessment.

The students were asked to produce videos under 3 minutes in length, but assessment takes longer than 3 minutes because we not only need to watch it, but also to write notes such that the marks can be explained to the students (see transparency above).

The time taken to perform the assessment was measured and it was found that a little under 10 minutes was required per video. This amount was reasonable, but it should be noted that it would be prohibitively difficult to ask one marker to assess a very large number of video assignments, and it would be significantly harder to maintain consistency between markers for a subjective task such as this, though no doubt it is possible to use techniques such as group testing to calibrate such marks.

\section{Statement of Ethics}

In the best of all possible worlds any experiment involving humans should seek ethics approval prior to being conducted, and ideally seek informed consent from participants.

However asking students to create videos was not intended as an experiment. It was intended as the best effort provide fair and effective assessment of students in a time of unprecedented difficulties.

The results of the exercise were so positive that I believe that the value of writing about this work outweighs any potential risk as those were very minor. Care has been taken to preserve anonymity of participants and all of the students who participated in AMP1 were provided with a draft of this report and given an opportunity to provide feedback or object to its publication.

\section{Conclusion}

In practice video assessment appeared to work very well. The students engaged with the task and produced excellent results. Many students enjoyed the course overall, and this was a significant part of that experience, which (at worst) did not detract from the experience.

A video assessment exercise requires support from teachers during the semester. This requires a significant amount of time and energy, and in future should involve providing some concrete feedback on "first draft" videos.

The exercise also requires time to assess ( 3 minute videos take almost 10 minutes each in order to view and then write feedback, and this should be doubled if early feedback is to be incorporated). This is significantly less time than required to assess written reports, but is not negligible.

The conclusion is that I believe we should use this approach more widely for small to medium classes, but it might be difficult to use for classes with some hundreds of students. Moreover the approach should be used in contexts where the students receive at least part of the content of the course in a format consistent with that asked from them.

Freedom of topic is an important component of this task and ideally when used in the future should be a core part of the task. At the very least it makes watching a large number of videos more interesting. More importantly it is a general principle that should be more often considered that students "buy into" tasks more when they have more control over the task, in this case over both topic and of presentation style.

A criticism of this approach is that students can focus on only one area of the course. Thus this type of assessment should be conducted together with other more traditional tasks.

Finally, although useful, the current "rubric" is rough. In the future I plan to create a more formal rubric with more detail, and a better ability to discriminate between the best students.

\section{ACKNOWLEDGEMENTS}

I would like to thank Melissa Humphries for valuable feedback on this work.

\section{REFERENCES}

Ale19. Bryan Alexander, Video assignments are the new term paper. how does that change teaching and learning?, Higher Education, Opinion, April 2019.

AMS17. Gender summary: Gender across the pipeline: unrealised potential, Australian Mathematical Sciences Institute, 2017, https://amsi.org.au/wp-content/uploads /2017/10/ dp2017-gender-summary3.pdf, accessed July 27th, 2020.

CAL19. Samantha Louise Clarke, Sue Atkinson, and Danny Liu, Making effective video assignments, July 2019, https:/ /educational-innovation.sydney.edu.au/ teaching@sydney/making-video-assignments/, accessed on July 17th, 2020.

FDWT16. Jessica K. Frawley, Laurel Evelyn Dyson, James Wakefield, and Jonathan Tyler, Supporting graduate attribute development in introductory accounting with student-generated screencasts, International Journal of Mobile and Blended Learning (IJMBL) (2016).

For20. ACM Presidential Task Force, Virtual conferences: A guide to best practices, May 2020, https://people. clarkson.edu/ jmatthew/acm/VirtualConferences_ GuideToBestPractices_CURRENT.pdf. 
Har78. Susan Harter, Pleasure derived from challenge and the effects of receiving grades on children's difficulty level choice, Child Development 49 (1978), 788-799.

HBR20. Rowena Harper, Tracey Bretag, and Kiata Rundle, Detecting contract cheating: examining the role of assessment type, Higher Education Research \& Development (2020), https://doi. org/10.1080/07294360.2020.1724899.

HCW10. Mark Huxham, Fiona Campbell, and Jenny Westwood, Oral versus written assessments: a test of student performance and attitudes, Assessment \& Evaluation in Higher Education 37 (2010), no. 1, 125-136.

ICR20. P. Iannone, C. Czichowsky, and J. Ruf, The impact of high stakes oral performance assessment on students' approaches to learning: a case study, Educ Stud Math 103 (2020), 313-337, https://doi.org/10.1007/s10649-020-09937-4.

Jen10. B. B. Jensen, Oral assessment in engineering education, The International Journal of Electrical Engineering and Education 47 (2010), no. 4, 375-379, https:/ / doi.org/10.7227/IJEEE. 47.4.2.

Koh99. Alfie Kohn, Punished by rewards, Mariner Books, 1999.

$\mathrm{SCA}^{+}$19. Shima Salehi, Sehoya Cotner, Samira M. Azarin, Erin E. Carlson, Michelle Driessen, Vivian E. Ferry, William Harcombe, Suzanne McGaugh, Deena Wassenberg, Azariah Yonas, and Cissy J. Ballen, Gender performance gaps across different assessment methods and the underlying mechanisms: The case of incoming preparation and test anxiety, Frontiers in Education 4 (2019).

SD20. Prométhée Spathis and Ratan Dey, What is Zoom not telling you: Lessons from an online courseduring COVID-19, ACM Sigcomm Education Workshop, August 2020, http:/ /gaia.cs.umass.edu/sigcomm_education_ workshop_2020/papers/sigcommedu20-final16.pdf.

wat20. Transitioning to online lectures, Centre for Teaching Excellence, University of Waterloo, 2020, https://uwaterloo. $\mathrm{ca} /$ centre-for-teaching-excellence/teaching-resources / teaching-tips/teaching-tips-educational-technologies/ transitioning-online-lectures, accessed August 6th, 2020. 